QUARTERLY OF APPLIED MATHEMATICS

VOLUME LXIX, NUMBER 2

JUNE 2011, PAGES 357-378

S $0033-569 X(2011) 01235-1$

Article electronically published on March 10, 2011

\title{
LACK OF CONTACT IN A LUBRICATED SYSTEM
}

\author{
BY \\ IONEL CIUPERCA (Université de Lyon, Université Lyon 1, CNRS, UMR 5208, Institut Camille \\ Jordan, Bat. Braconnier, 43, blvd du 11 novembre 1918, F-69622 Villeurbanne Cedex, France) \\ AND \\ J. IGNACiO TELlo (Matemática Aplicada, E.U.I. Informática, Universidad Politécnica de Madrid, \\ 28031 Madrid, Spain)
}

\begin{abstract}
We consider the problem of a rigid surface moving over a flat plane. The surfaces are separated by a small gap filled by a lubricant fluid. The relative position of the surfaces is unknown except for the initial time $t=0$. The total load applied over the upper surface is a known constant for $t>0$. The mathematical model consists of a coupled system formed by the Reynolds variational inequality for incompressible fluids and Newton's Second Law. We study the steady states of the problem and the global existence and uniqueness of the time-dependent problem. We assume one degree of freedom for the position of the surface. We consider different cases depending on the geometry of the upper surface.
\end{abstract}

1. Introduction. Lubricated contacts are widely used in mechanical systems to connect solid bodies in relative motion. A lubricant fluid is introduced in the narrow space between the bodies with the purpose of avoiding direct solid-to-solid contact.

This contact is said to be in the hydrodynamic regime, and the forces transmitted between the bodies result from the shear and pressure forces developed in the lubricant film.

We consider one of the simplest lubricated systems, which consists of two rigid surfaces in hydrodynamic contact. The bottom surface, assumed planar and horizontal, moves with a constant horizontal translation velocity, and a vertical given force $F>0$ is applied vertically on the upper body.

Received November 20, 2009.

2000 Mathematics Subject Classification. Primary 35J20, 47H11, 49J10; Secondary 76D08.

Key words and phrases. Lubricated systems, Reynolds variational inequality, global solutions, stationary solutions.

The second author was partially supported by project MTM2009-13655 Ministerio de Ciencia e Innovación (Spain).

E-mail address: ciuperca@math.univ-lyon1.fr

E-mail address: jtello@eui.upm.es 
The wedge between the two surfaces is filled with an incompressible fluid. We suppose that the wedge satisfies the thin-film hypothesis, so that a Reynolds-type model can be used to describe the problem.

We denote by $\Omega$ the two-dimensional domain in which the hydrodynamic contact occurs. We assume that $\Omega$ is open, bounded and with regular boundary $\partial \Omega$. Without loss of generality we consider $0 \in \Omega$. We assume that the upper body, the slider, is allowed to move only by vertical translation. The normalized distance between the surfaces is given by

$$
h(x, t)=h_{0}(x)+\eta(t),
$$

where $\eta(t)>0$ represents the vertical translation of the slider and $h_{0}: \Omega \longrightarrow[0, \infty[$ describes the shape of the slider and is a given function satisfying

$$
h_{0} \in C^{0}(\bar{\Omega}), \quad \min _{x \in \Omega} h_{0}(x)=h_{0}(0)=0 .
$$

The mathematical model we study considers the possible cavitation in the thin film, so the (normalized) pressure " $p$ " of the fluid satisfies the Reynolds variational inequality (see [8]):

$$
\int_{\Omega} h^{3} \nabla p \cdot \nabla(\varphi-p) \geq \int_{\Omega} h \frac{\partial}{\partial x_{1}}(\varphi-p)-\eta^{\prime}(t) \int_{\Omega}(\varphi-p), \quad \forall \varphi \in K,
$$

where

$$
K=\left\{\varphi \in H_{0}^{1}(\Omega): \varphi \geq 0\right\}
$$

and " $\nabla$ " denotes the gradient with respect to the variables $x \in \Omega$. Without loss of generality we assume the velocity of the bottom surface is oriented in the direction of the $x_{1}$-axis and its normalized value is equal to 1 .

The equation of motion of the slider is

$$
\eta^{\prime \prime}=\int_{\Omega} p d x-F \quad \text { (second Newton Law) }
$$

completed with the initial conditions:

$$
\begin{gathered}
\eta(0)=\eta_{1}^{0}, \\
\eta^{\prime}(0)=\eta_{2}^{0},
\end{gathered}
$$

where $\eta_{1}^{0}>0, \eta_{2}^{0} \in \mathbb{R}$ are given data.

The unknowns of the problem are the pressure $p(x, t)$ and the vertical displacement of the slider $\eta(t)$. It is known that for any given $C^{1}$ function $\eta(t)$ the problem (1.2) is well posed (see for instance [12]). The system (1.2)-(1.5) is equivalent to the following Cauchy problem for a second-order ordinary differential equation in $\eta$ :

$$
\left\{\begin{array}{l}
\eta^{\prime \prime}=G\left(\eta, \eta^{\prime}\right), \\
\eta(0)=\eta_{1}^{0}, \\
\eta^{\prime}(0)=\eta_{2}^{0},
\end{array}\right.
$$


where $G:] 0, \infty[\times \mathbb{R} \longrightarrow \mathbb{R}$ is given by

$$
G(\beta, \gamma):=\int_{\Omega} q(x) d x-F,
$$

and $q \in K$ (depending on $\beta$ and $\gamma$ ) is the unique solution to

$$
\left\{\begin{array}{l}
\int_{\Omega}\left(h_{0}+\beta\right)^{3} \nabla q \cdot \nabla(\varphi-q) \geq \int_{\Omega} h_{0} \frac{\partial}{\partial x_{1}}(\varphi-q)-\gamma \int_{\Omega}(\varphi-q), \\
\forall \varphi \in K .
\end{array}\right.
$$

The main goal of the paper is to give sufficient conditions for the shape $h_{0}$ of the slider to obtain global existence of solutions to (1.6); i.e., there is no contact solid-to-solid for $t<\infty$. We also study the existence of steady states of the problem. Another interesting physical question which we adress here is to see if there exists a "barrier" value $\eta_{b}>0$ such that $\eta(t) \geq \eta_{b}$ for all $t>0$.

We prove the existence of $\eta_{b}$ for two of the three cases studied. In the third case (the so-called "flat case"), we prove that $\eta$ tends to 0 as $t \rightarrow \infty$.

The main idea of the proofs of these results is to find a lower bound of the force exerted by the pressure of the fluid on the upper body when the distance between the surfaces decreases (i.e., $\left.\eta^{\prime} \leq 0\right)$. This lower bound admits an expression of the form $F_{S}+F_{D}$, where $F_{S}$ is a "spring-like" force and $F_{D}$ is a "damping force" (see Corollary (3.2) and Remark (3.3)).

$F_{S}$ depends only on the position $\eta(t)$ and represents the force exerted by the pressure of the fluid for the stationary position in an auxiliary subdomain $U$ of $\Omega$.

$F_{D}$ is of the form $F_{D}=-\eta^{\prime} d$, where $d$ is a "damping" coefficient and depends only on $\eta$. The global existence of the solution $\eta$ is a consequence of the high velocity of blowup of $d$ when $\eta$ tends to 0 . The existence of a "barrier" $\eta_{b}$ and the steady states is based on the fact that $F_{S}$ blows up when $\eta$ tends to 0 . In the "flat case", the force $F_{S}$ is equal to zero, which explains the nonexistence of a barrier and also the nonexistence of stationary solutions in this case.

We also prove that when the velocity of blowup for $d$ and $F_{S}$ is not large enough, when $\eta \rightarrow 0$ there exist initial data for which the solution $\eta$ is not global in time.

The present work is related to different articles on the fluid-rigid interaction problems (see, for example, [5], 6], [9, [10] and [11, for a nonexhaustive bibliography on this subject). These papers concern the study of the motion of one or many rigid bodies inside a domain $Q \in \mathbb{R}^{n}, n=2,3$, filled with an incompressible fluid with constant viscosity. The mathematical model is a coupled system between Navier-Stokes equations modeling the fluid and the second Newton Law to describe the rigid bodies' positions. A relevant problem in this context is the so-called "noncollision" problem, where the question is to know if this body will touch the boundary $\partial Q$ of the fluid in finite time.

In [11, Hillairet considered the particular case where $Q$ is the half-plane $\mathbb{R} \times \mathbb{R}_{+}$and the rigid body is a disk which moves only along the vertical axis. He proves that in the absence of external forces the solution is defined globally in time. He also shows that the disk always remains "far" from the boundary. 
In [9], Gérard-Varet and Hillairet consider a more general shape of the rigid body in a general domain $Q$ in the presence of gravity. They assume Hölder regularity $C^{1, \alpha}$ (for $0<\alpha \leq 1$ ) of the boundary of the rigid body and of $Q$. They prove global existence in time if and only if $\alpha \geq \frac{1}{2}$, which emphasizes the role of roughness in the collision scenario; when the solution is global, the distance between the rigid body and the boundary of $Q$ can go to zero when time goes to infinity. Similar results are given by Hesla in [10].

The main difference between the above-mentioned works and the present one is the obtention in this study of a "barrier" value $\eta_{b}>0$ and also of a stationary solution for any exterior force $F$. We can explain this difference by the high shear and pressure that develop in a lubricant fluid film, due especially to the relative motion of the closed surfaces. An interesting open question is to see if similar "barrier" results and steady state solutions can be obtained for situations when the thin film hypothesis is not satisfied in the fluid (so the full Navier-Stokes equations must be used in the place of Reynolds models), but relative horizontal motion exists between the two surfaces.

Fluid-rigid interaction problems in lubrication were also considered in [7, where the Reynolds equation is used in the place of the Reynolds variational inequality in the particular "flat" case. We also mention the papers [1, 2] and [4, where the existence of steady states is studied for lubricated systems with two degrees of freedom.

The contents of the paper are the following:

In Section 2 we present the main results concerning existence of global solutions for the time-dependent problem and existence of steady state solutions. We also present the assumptions needed for $h_{0}$. In Section 3 we prove some properties of the function $G$ (the balance of forces). Section 4 is devoted to the proof of the main results presented in Section 2. In Section 5 we present some examples for which solutions do not exist globally in time.

2. Main results. We begin by the local in time existence and uniqueness result, for which the minimal hypothesis (1.1) is sufficient. The result is enclosed in the following theorem and it is a consequence of the regularity of $G$ (see Lemma 3.4).

Theorem 2.1. The problem (1.6) has a unique solution locally in time.

Let $\left[0, T\left[\right.\right.$ be the maximal interval of existence of a solution to (1.6), so $\eta \in C^{2}([0, T[)$. The main goal of the paper is to give conditions which guaranty global existence (i.e. $T=+\infty)$. It is equivalent to prove that for any fixed $T>0$ there exist $m>0$ and $M>0$ (depending eventually on $T$ ) such that

$$
\left\{\begin{array}{l}
m \leq \eta(t) \leq M, \quad \text { for all } t \in[0, T[ \\
\left|\eta^{\prime}(t)\right| \leq M
\end{array}\right.
$$

Moreover, we are interested in knowing if there exist such constants $m$ and $M$ independent of $T$.

We consider three different cases depending on the shape of the slide $h_{0}$.

CASE I (Line contact). We assume that $h$ is equal to 0 only in the line $\left\{x_{1}=0\right\}$, i.e.

$$
\left\{\begin{array}{l}
h_{0}\left(0, x_{2}\right)=0 \text { for all } x_{2} \in \mathbb{R} \text { such that }\left(0, x_{2}\right) \in \Omega \text { and } \\
h_{0}\left(x_{1}, x_{2}\right)>0 \text { for all }\left(x_{1}, x_{2}\right) \in \Omega, x_{1} \neq 0 .
\end{array}\right.
$$


We also assume that there exists $\alpha>0$ such that

$$
h_{0}\left(x_{1}, x_{2}\right) \sim\left|x_{1}\right|^{\alpha} \text { when } x_{1} \rightarrow 0 .
$$

More precisely there exist a neighborhood $W$ of 0 and a function $h_{1}$ regular enough on the closure $\bar{W}$ of $W$ with $h_{1}>0$ on $\bar{W}$ such that

$$
h_{0}\left(x_{1}, x_{2}\right)=\left|x_{1}\right|^{\alpha} h_{1}\left(x_{1}, x_{2}\right) \text { in } W .
$$

CASE II (Point contact). We assume that $h_{0}$ is equal to 0 only in the point $\{x=0\}$, i.e.

$$
h_{0}(0)=0 \text { and } h_{0}(x)>0 \text { for all } x \in \Omega-\{0\} .
$$

We also assume that there exists $\alpha>0$ such that

$$
h_{0}(x) \sim|x|^{\alpha} \text { when } x \rightarrow 0 ;
$$

that is, there exist $W$ and $h_{1}$ as in Case I such that

$$
h_{0}(x)=|x|^{\alpha} h_{1}(x) \text { in } W
$$

(where $|\cdot|$ is the Euclidean norm in $\mathbb{R}^{2}$ ).

CASE III (Flat slides). We assume that $h$ is flat, i.e.

$$
h_{0}=0 \quad \text { on } \Omega \text {, }
$$

which implies $h(x, t)=\eta(t)$.

Results of global existence and barrier functions are presented in the following theorem:

Theorem 2.2. We assume that $h_{0}$ satisfies assumption (2.2) in case I or (2.3) in case II for $\alpha$ satisfying

$$
\left\{\begin{array}{l}
\alpha \geq \frac{3}{2} \quad \text { in Case I (line contact), } \\
\alpha \geq 2 \quad \text { in Case II (point contact). }
\end{array}\right.
$$

Then $T=+\infty$. Moreover, there exist constants $\eta_{b}, M_{0}$ and $M_{1}$ such that $0<\eta_{b} \leq M_{0}$ and $M_{1} \geq 0$ satisfy:

$$
\left\{\begin{array}{l}
\eta_{b} \leq \eta(t) \leq M_{0} \\
\left|\eta^{\prime}(t)\right| \leq M_{1}
\end{array}\right.
$$

for $t \geq 0$.

REMARK 2.3. In section 5 we prove that for any $\alpha<\frac{3}{2}$ in case I and $\alpha<2$ in case II there are examples of $\Omega$ and $h_{0}$ for which the problem does not admit global solutions $(T<\infty)$. This shows that the results of Theorem 2.2 are optimal.

REMARK 2.4. For the one-dimensional problem, i.e. $\Omega$ is an interval of $\mathbb{R}$, the results are the same as in case I (line contact) for the two-dimensional problem.

Theorem 2.5. Under the hypothesis $h_{0} \equiv 0$ (Case III) we have $T=+\infty$. Moreover there exist $M_{0}, M_{1}>0$ such that

$$
\left\{\begin{array}{l}
0<\eta(t) \leq M_{0} \\
\left|\eta^{\prime}(t)\right| \leq M_{1}
\end{array}\right.
$$


for $t \geq 0$ and

$$
\eta(t) \rightarrow 0 \quad \text { as } \quad t \rightarrow+\infty .
$$

Moreover there exist $t_{0} \geq 0, a>0$ and $b_{1}<b_{2} \in \mathbb{R}$ with $t_{0}+b_{1}>0$ such that

$$
\frac{a}{\sqrt{t+b_{2}}} \leq \eta(t) \leq \frac{a}{\sqrt{t+b_{1}}} \quad \forall t \geq t_{0}
$$

In addition, stationary solutions do not exist for the system (1.6).

REMARK 2.6. The same result can be obtained for the corresponding one-dimensional problem.

The results concerning the existence of steady states for cases I and II are enclosed in the following theorem:

Theorem 2.7. Let $h_{0}$ satisfy assumption (2.2) in case I or (2.3) in case II for $\alpha$ satisfying

$$
\left\{\begin{array}{l}
\alpha \geq 1 \quad \text { in Case I (line contact), } \\
\alpha>\frac{3}{2} \quad \text { in Case II (point contact). }
\end{array}\right.
$$

Then there exists at least one stationary solution $\bar{\eta}>0$ of the Cauchy problem (1.6), i.e.

$$
G(\bar{\eta}, 0)=0 .
$$

REmark 2.8. When $F$ is large enough, for $\alpha<1$ (case I) and $\alpha<\frac{4}{3}$ (case II) stationary solutions do not exist. This is a consequence of the fact that

$$
\lim _{\eta \rightarrow 0^{+}} G(\eta, 0)<\infty
$$

(see [3]). In case II for $\alpha \in\left[\frac{4}{3}, \frac{3}{2}\right]$ the problem remains open for general situations.

REMARK 2.9. The uniqueness of the stationary solution for the 1-dimensional problem was proved in 2 for a particular function $h_{0}$. The uniqueness in the general case remains open, as well as the existence of periodic solutions or the asymptotic behavior of the solutions of the time-dependent problem (1.6).

\section{Some preliminary results on the function $G$.}

3.1. General properties of $G$. In this subsection we prove some preliminary results on $G$ under the minimal assumption (1.1).

Let $V_{1}$ be defined as follows:

$$
V_{1}=\sup _{x \in \Omega}\left\{-\frac{\partial h_{0}}{\partial x_{1}}(x)\right\}
$$

Notice that $V_{1} \geq 0$.

Lemma 3.1. i) There exists $c_{1}>0$ such that

$$
G(\beta, \gamma) \leq \frac{c_{1}}{\beta^{3}}-F \quad \forall \beta>0, \gamma \geq 0 .
$$

ii)

$$
G(\beta, \gamma)=-F \quad \forall \beta>0, \gamma \geq V_{1} .
$$


Proof. i) We take $\varphi=0$ and $\varphi=2 q$ in (1.7) to have

$$
\int_{\Omega}\left(h_{0}+\beta\right)^{3}|\nabla q|^{2}=\int_{\Omega} h_{0} \frac{\partial q}{\partial x_{1}}-\gamma \int_{\Omega} q .
$$

We use the inequalities $h_{0}+\beta \geq \beta, \gamma \geq 0, q \geq 0$ to obtain

$$
\beta^{3} \int_{\Omega}|\nabla q|^{2} \leq \int_{\Omega} h_{0} \frac{\partial q}{\partial x_{1}} .
$$

We use the Poincaré inequality and the proof of case i) ends.

ii) The inequality (1.7) can be written as

$$
\int_{\Omega}\left(h_{0}+\beta\right)^{3} \nabla q \cdot \nabla(\varphi-q) \geq-\int_{\Omega}\left(\frac{\partial h_{0}}{\partial x_{1}}+\gamma\right)(\varphi-q), \quad \forall \varphi \in K .
$$

Since

$$
\gamma+\frac{\partial h_{0}}{\partial x_{1}} \geq 0, \quad \forall x \in \Omega
$$

we have that $q=0$, which gives the result.

Lemma 3.2. Let $U \subset \Omega$ be an arbitrary open set. Consider $\beta>0$ and $q_{1 \beta}, q_{2 \beta}$ the solutions to the problems

$$
\begin{cases}-\nabla \cdot\left[\left(h_{0}+\beta\right)^{3} \nabla q_{1 \beta}\right]=-\frac{\partial h_{0}}{\partial x_{1}} & \text { on } U, \\ q_{1 \beta}=0 & \text { on } \partial U,\end{cases}
$$

and

$$
\begin{cases}-\nabla \cdot\left[\left(h_{0}+\beta\right)^{3} \nabla q_{2 \beta}\right]=1 & \text { on } U, \\ q_{2 \beta}=0 & \text { on } \partial U,\end{cases}
$$

respectively. We then have

$$
G(\beta, \gamma) \geq \int_{U} q_{1 \beta} d x-\gamma \int_{U} q_{2 \beta} d x-F \quad \text { for } \gamma \in \mathbb{R} .
$$

Proof. By linearity of (3.2), (3.3) it is clear that $\hat{q}:=q_{1, \beta}-\gamma q_{2, \beta}$ is the solution to

$$
\begin{cases}-\nabla \cdot\left[\left(h_{0}+\beta\right)^{3} \nabla \hat{q}\right]=-\frac{\partial h_{0}}{\partial x_{1}}-\gamma & \text { on } U \\ \hat{q}=0 & \text { on } \partial U .\end{cases}
$$

Now, $\left.q\right|_{U}$ is a super solution of the problem (3.5). By the maximum principle we have $q \geq \hat{q}$, which proves the lemma.

Remark 3.3. The expressions $\int_{U} q_{1 \beta}$ and $\int_{U} q_{2 \beta}$ represent the force " $F_{S}$ " and the damping coefficient " $d$ ", respectively, as we described in the introduction.

Lemma 3.4. The function $G$ is locally lipschitzian.

Proof. Let us consider $S$ to be a compact set included in $] 0, \infty[\times \mathbb{R}$, and take $(\beta, \gamma)$ and $(\tilde{\beta}, \tilde{\gamma})$ in $S$. We denote by $\tilde{q} \in K$ the solution to the Reynolds inequality

$$
\int_{\Omega}\left(h_{0}+\tilde{\beta}\right)^{3} \nabla \tilde{q} \cdot \nabla(\varphi-\tilde{q}) \geq \int_{\Omega} h_{0} \frac{\partial}{\partial x_{1}}(\varphi-\tilde{q})-\tilde{\gamma} \int_{\Omega}(\varphi-\tilde{q}), \quad \forall \varphi \in K .
$$


We take $\varphi=\tilde{q}$ in (1.7), $\varphi=q$ in (3.6) and we add both inequalities to get, after elementary computations,

$$
\begin{aligned}
\int_{\Omega}\left(h_{0}+\right. & \beta)^{3}|\nabla(\tilde{q}-q)|^{2} \\
& \leq|\tilde{\gamma}-\gamma||\Omega|^{1 / 2}\|\tilde{q}-q\|_{L^{2}(\Omega)}+|\tilde{\beta}-\beta|\left\|A_{\beta, \tilde{\beta}}\right\|_{L^{\infty}}\|\nabla \tilde{q}\|_{L^{2}(\Omega)}\|\nabla(\tilde{q}-q)\|_{L^{2}(\Omega)},
\end{aligned}
$$

where $A_{\beta, \tilde{\beta}}=\left(h_{0}+\beta\right)^{2}+\left(h_{0}+\tilde{\beta}\right)^{2}+\left(h_{0}+\beta\right)\left(h_{0}+\tilde{\beta}\right)$. By the Poincaré inequality, the proof ends.

3.2. The case of a nonhorizontal slider. In this subsection we assume $h_{0} \neq 0$ and also that $h_{0}$ satisfies the hypotheses of Cases I or II (line contact and point contact cases respectively). We also assume that $\alpha>1$ in this subsection except when it is stated to the contrary. We prove the existence of a subdomain $U \subset \Omega$ such that the averages of the corresponding functions $q_{1 \beta}$ and $q_{2 \beta}$ are "large" in some sense when $\beta$ is small.

We denote by $\rho \geq 0$ and $\theta \in[0,2 \pi]$ the polar coordinates of $\left(x_{1}, x_{2}\right)$.

Lemma 3.5. a) Case I. (Line contact)

There exist $\delta, \beta_{0}, c_{2}>0$ and $B_{l, \beta}$ defined by

$$
\left.B_{l, \beta}:=\right]-2 \beta^{1 / \alpha},-\beta^{1 / \alpha}[\times]-\delta, \delta[
$$

such that for any $0<\beta \leq \beta_{0}$ we have

$$
\frac{\partial h_{0}}{\partial x_{1}} \leq-c_{2} \beta^{1-1 / \alpha} \quad \text { on } B_{l, \beta} .
$$

b) Case II. (Contact point)

There exist $\left.\theta_{0} \in\right] 0, \frac{\pi}{2}\left[, c_{2}, \beta_{0}>0\right.$ and the sector $B_{p, \beta}$ defined by

$$
B_{p, \beta}=\left\{\left(x_{1}, x_{2}\right) \in \mathbb{R}^{2} ; \beta^{1 / \alpha} \leq \rho \leq 2 \beta^{1 / \alpha} ; \pi-\theta_{0} \leq \theta \leq \pi+\theta_{0}\right\}
$$

such that for any $0<\beta \leq \beta_{0}$ :

$$
\frac{\partial h_{0}}{\partial x_{1}} \leq-c_{2} \beta^{1-1 / \alpha} \quad \text { on } B_{p, \beta} .
$$

Proof. a) We have for $x_{1} \leq 0$,

$$
\frac{\partial h_{0}}{\partial x_{1}}=-\alpha\left(-x_{1}\right)^{\alpha-1} h_{1}+\left(-x_{1}\right)^{\alpha} \frac{\partial h_{1}}{\partial x_{1}}=\left(-x_{1}\right)^{\alpha-1} h_{1}(x)\left[-\alpha-x_{1} \frac{\frac{\partial h_{1}}{\partial x_{1}}}{h_{1}(x)}\right] .
$$

Since $h_{1}>0$ on $\bar{W}$ we obtain

$$
x_{1} \frac{\frac{\partial h_{1}}{\partial x_{1}}}{h_{1}(x)} \longrightarrow 0 \quad \text { when } x \longrightarrow 0,
$$

and the result is obvious.

b) For any $x$ in $W-\{0\}$ we have

$$
\frac{\partial h_{0}}{\partial x_{1}}=\alpha|x|^{\alpha-1} \frac{x_{1}}{|x|} h_{1}+|x|^{\alpha} \frac{\partial h_{1}}{\partial x_{1}}=|x|^{\alpha-1} h_{1}\left(\alpha \frac{x_{1}}{|x|}+|x| \frac{\partial h_{1}}{h_{1}}\right) .
$$


Now we can choose $\left.\theta_{0} \in\right] 0, \frac{\pi}{2}$ [ such that $\frac{x_{1}}{|x|}<-\frac{1}{2}$ if $\pi-\theta_{0} \leq \theta \leq \pi+\theta_{0}$ (choose for example $\left.\theta_{0}=\frac{\pi}{6}\right)$. On the other hand, we have

$$
|x| \frac{\frac{\partial h_{1}}{\partial x_{1}}}{h_{1}} \longrightarrow 0 \quad \text { when } \quad x \longrightarrow 0,
$$

which proves the lemma.

LEMma 3.6. Let us consider $q_{1 \beta}, q_{2 \beta}$ to be the solutions to (3.2)-(3.3), where $U$ is given by

$$
U:=B_{l, \beta} \quad \text { in case I, } \quad U:=B_{p, \beta} \quad \text { in case II, }
$$

with $B_{l, \beta}$ and $B_{p, \beta}$ defined in Lemma 3.5. Then there exist $\beta_{0}, c_{3}, c_{4}>0$ such that for any $\beta \in] 0, \beta_{0}$ ] we obtain

$$
\begin{cases}\int_{B_{l, \beta}} q_{1 \beta} \geq c_{3} \beta^{2(1 / \alpha-1)} & \text { in case I, } \\ \int_{B_{p, \beta}} q_{1 \beta} \geq c_{3} \beta^{3 / \alpha-2} & \text { in case II, }\end{cases}
$$

and

$$
\begin{cases}\int_{B_{l, \beta}} q_{2 \beta} \geq c_{4} \beta^{3(1 / \alpha-1)} & \text { in case I, } \\ \int_{B_{p, \beta}} q_{2 \beta} \geq c_{4} \beta^{4 / \alpha-3} & \text { in case II. }\end{cases}
$$

Proof. From (3.3) we deduce

$$
\int_{U} q_{2 \beta}=\int_{U}\left(h_{0}+\beta\right)^{3}\left|\nabla q_{2 \beta}\right|^{2} .
$$

From the equality

$$
\int_{U}\left(h_{0}+\beta\right)^{3} \nabla q_{2 \beta} \cdot \nabla \varphi=\int_{U} \varphi, \quad \text { for all } \varphi \in H_{0}^{1}(U)
$$

and the Cauchy-Schwarz inequality, we get

$$
\int_{U}\left(h_{0}+\beta\right)^{3}\left|\nabla q_{2 \beta}\right|^{2} \geq \sup _{\varphi \in H_{0}^{1}(U), \varphi \neq 0}\left[\frac{\left(\int_{U} \varphi\right)^{2}}{\int_{U}\left(h_{0}+\beta\right)^{3}|\nabla \varphi|^{2}}\right] .
$$

It suffices to find appropriate test functions $\varphi \in H_{0}^{1}(U), \varphi \neq 0$ such that the term

$$
\frac{\left(\int_{U} \varphi\right)^{2}}{\int_{U}\left(h_{0}+\beta\right)^{3}|\nabla \varphi|^{2}}
$$

is large enough.

Proof of (3.8).

CASE I (Line contact). We choose

$$
\varphi\left(x_{1}, x_{2}\right)=\psi_{1}\left(\frac{x_{1}}{\beta^{1 / \alpha}}\right) \psi_{2}\left(x_{2}\right)
$$

with $\psi_{1} \in \mathcal{D}(]-2,-1[), \psi_{1} \geq 0, \psi_{1} \not \equiv 0$ and $\psi_{2} \in D(]-\delta_{2}, \delta_{2}[), \psi_{2} \geq 0, \psi_{2} \not \equiv 0$. Then

$$
\int_{B_{l, \beta}} \varphi=\int_{-2 \beta^{1 / \alpha}}^{-\beta^{1 / \alpha}} \int_{-\delta_{2}}^{\delta_{2}} \psi_{1}\left(\frac{x_{1}}{\beta^{1 / \alpha}}\right) \psi_{2}\left(x_{2}\right) d x_{1} d x_{2},
$$


SO

$$
\int_{B_{l, \beta}} \varphi=\beta^{1 / \alpha} \int_{-2}^{-1} \psi_{1}\left(y_{1}\right) d y_{1} \int_{-\delta_{2}}^{\delta_{2}} \psi_{2}\left(x_{2}\right) d x_{2} .
$$

On the other hand, we have

$$
\begin{aligned}
& \int_{B_{l, \beta}}\left(h_{0}+\beta\right)^{3}|\nabla \varphi|^{2} \\
& \quad=\int_{-2 \beta^{1 / \alpha}}^{-\beta^{1 / \alpha}} \int_{-\delta_{2}}^{\delta_{2}}\left(h_{0}+\beta\right)^{3}\left[\frac{1}{\beta^{2 / \alpha}}\left|\psi_{1}^{\prime}\left(\frac{x_{1}}{\beta^{1 / \alpha}}\right) \psi_{2}\left(x_{2}\right)\right|^{2}+\left|\psi_{1}\left(\frac{x_{1}}{\beta^{1 / \alpha}}\right) \psi_{2}^{\prime}\left(x_{2}\right)\right|^{2}\right] d x_{1} d x_{2} .
\end{aligned}
$$

It is easy to show that

$$
\int_{B_{l, \beta}}\left(h_{0}+\beta\right)^{3}|\nabla \varphi|^{2} \leq c_{2}^{\prime} \beta^{3-1 / \alpha},
$$

where $c_{2}^{\prime}>0$ is a constant independent of $\beta$. From (3.10) for $U=B_{l, \beta}$ and (2.1), (3.12), we deduce

$$
\int_{B_{l, \beta}}\left(h_{0}+\beta\right)^{3}\left|\nabla q_{2 \beta}\right|^{2} \geq c_{3} \beta^{3(1 / \alpha-1)}
$$

and thanks to (3.9) we obtain $(3.8)_{1}$.

CASE II (Contact point). We choose $\varphi\left(x_{1}, x_{2}\right)=\psi_{3}\left(\frac{\rho}{\beta^{1 / \alpha}}\right) \psi_{4}(\theta)$ with $\psi_{3} \in \mathcal{D}(] 1,2[)$, $\psi_{3} \geq 0, \psi_{3} \not \equiv 0$, and $\psi_{4} \in \mathcal{D}(] \pi-\theta_{0}, \pi+\theta_{0}[), \psi_{4} \geq 0, \psi_{4} \not \equiv 0$. In polar coordinates, we have

$$
\begin{aligned}
\int_{B_{p, \beta}} \varphi & =\int_{\beta^{1 / \alpha}}^{2 \beta^{1 / \alpha}} \int_{\pi-\theta_{0}}^{\pi+\theta_{0}} \psi_{3}\left(\frac{\rho}{\beta^{1 / \alpha}}\right) \psi_{4}(\theta) \rho d \rho d \theta \\
& =\beta^{2 / \alpha} \int_{1}^{2} \psi_{3}\left(\rho_{1}\right) \rho_{1} d \rho_{1} \int_{\pi-\theta_{0}}^{\pi+\theta_{0}} \psi_{4}(\theta) d \theta
\end{aligned}
$$

and

$$
\begin{aligned}
& \int_{B_{p, \beta}}\left(h_{0}+\beta\right)^{3}|\nabla \varphi|^{2} \\
& \quad=\int_{\hat{B}_{\rho, \beta}}\left(h_{0}+\beta\right)^{3}\left[\frac{1}{\beta^{2 / \alpha}}\left|\psi_{3}^{\prime}\left(\frac{\rho}{\beta^{1 / \alpha}}\right)\right|^{2}\left|\psi_{4}(\theta)\right|^{2}+\frac{1}{\rho^{2}}\left|\psi_{3}\left(\frac{\rho}{\beta^{1 / \alpha}}\right)\right|^{2}\left|\psi_{4}^{\prime}(\theta)\right|^{2}\right] \rho d \theta d \rho,
\end{aligned}
$$

where $\hat{B}_{\rho, \beta}:=\left[\beta^{\frac{1}{\alpha}}, 2 \beta^{\frac{1}{\alpha}}\right] \times\left[\pi-\theta_{0}, \pi+\theta_{0}\right]$. We easily obtain

$$
\int_{B_{p, \beta}}\left(h_{0}+\beta\right)^{3}|\nabla \varphi|^{2} \leq c_{2}^{\prime} \beta^{3},
$$

where $c_{2}^{\prime}>0$ is a constant independent of $\beta$. From (3.10) we obtain

$$
\int_{B_{p, \beta}}\left(h_{0}+\beta\right)^{3}\left|\nabla q_{2 \beta}\right|^{2} \geq c_{3} \beta^{4 / \alpha-3},
$$

and by $(3.9)$ we get $(3.8) 2$.

Proof of (3.7). From Lemma 3.5 we have

$$
-\frac{\partial h_{0}}{\partial x_{1}} \geq c_{2} \beta^{1-1 / \alpha} \quad \text { on } B_{l, \beta} \quad \text { (in case I) }
$$


and

$$
-\frac{\partial h_{0}}{\partial x_{1}} \geq c_{2} \beta^{1-1 / \alpha} \quad \text { on } B_{p, \beta} \quad \text { (in case II). }
$$

By the maximum principle we deduce the inequality

$$
q_{1 \beta} \geq c_{2} \beta^{1-1 / \alpha} q_{2 \beta} \quad \text { on } B_{l, \beta} \quad \text { (respectively } B_{p, \beta} \text { ) }
$$

and by (3.8) the proof ends.

The following lemma gives lower bounds for the function $G$.

Lemma 3.7. There exist $\beta_{0}, c_{3}, c_{4}$ such that for any $\left.\left.\beta \in\right] 0, \beta_{0}\right]$ and $\gamma \leq 0$ we have

$$
\begin{gathered}
G(\beta, \gamma) \geq c_{3} \beta^{2(1 / \alpha-1)}-\gamma c_{4} \beta^{3(1 / \alpha-1)}-F, \quad \text { for } \alpha>1 \text { in case I, } \\
G(\beta, \gamma) \geq c_{3} \log \frac{1}{\beta}-\gamma c_{4} \log \frac{1}{\beta}-F, \quad \text { for } \alpha=1 \text { in case I, }
\end{gathered}
$$

or

$$
G(\beta, \gamma) \geq c_{3} \beta^{3 / \alpha-2}-\gamma c_{4} \beta^{4 / \alpha-3}-F, \quad \text { for } \alpha>\frac{3}{2} \text { in case II. }
$$

Proof. (3.13) and (3.15) are consequences of Lemma 3.2 and Lemma 3.6 The inequality (3.14) cannot be obtained in the same fashion. It is a consequence of Lemma 4.2 and Theorem 3.7 of 3 .

\section{Proof of the main results.}

4.1. Proof of Theorem 2.2. In this subsection we assume that $h_{0}$ satisfies the hypotheses of Cases I or II (line contact and point contact case respectively). We consider $\eta(t)$ to be the solution of the Cauchy problem (1.6) defined on the maximal interval $[0, T[$. We prove that $\eta$ and $\eta^{\prime}$ are bounded and $\eta$ stays "far" from 0 . We first prove in Proposition 4.1 that $\eta^{\prime}$ admits an upper bound and the same for $\eta$ in Proposition 4.3 These results are needed to prove the existence of lower bounds for $\eta^{\prime}$ (Proposition 4.4) and $\eta$ (Proposition 4.5).

Let $V_{2}$ be defined by

$$
V_{2}=\max \left\{\eta_{2}^{0}+1, V_{1}\right\}
$$

for $V_{1}$ as in (3.1). Then we have:

Proposition 4.1.

$$
\eta^{\prime}(t)<V_{2} \quad \forall t \in[0, T[.
$$

Proof. Suppose the result is false and denote by $t_{1}>0$ the first point such that $\eta^{\prime}\left(t_{1}\right)=V_{2}$. This implies $\eta^{\prime \prime}\left(t_{1}\right) \geq 0$, which contradicts Lemma $\left.3.1 i i\right)$ where $\eta^{\prime \prime}\left(t_{1}\right)=$ $-F$.

We introduce two energies $\left.E_{1}, E_{2}:\right] 0,+\infty[\times \mathbb{R} \rightarrow \mathbb{R}$ defined by

$$
E_{1}(\beta, \gamma)=\frac{1}{2} \gamma^{2}+F \beta
$$

and

for $c_{1}$ as in Lemma 3.1 .

$$
E_{2}(\beta, \gamma)=\frac{1}{2} \gamma^{2}+F \beta+\frac{c_{1}}{2 \beta^{2}}
$$


The energies $E_{1}$ and $E_{2}$ are used in the following lemma when $\eta(t)$ is nonincreasing or nondecreasing respectively.

Lemma 4.2. For any $t \in[0, T[$ we have

i)

ii)

$$
\frac{d}{d t} E_{1}\left(\eta(t), \eta^{\prime}(t)\right) \leq 0 \quad \text { if } \quad \eta^{\prime}(t) \leq 0
$$

$$
\frac{d}{d t} E_{2}\left(\eta(t), \eta^{\prime}(t)\right) \leq 0 \quad \text { if } \quad \eta^{\prime}(t) \geq 0 .
$$

Proof. i) We multiply the equation

$$
\eta^{\prime \prime}+F=G\left(\eta, \eta^{\prime}\right)+F
$$

by $\eta^{\prime}$ and use the inequality $G\left(\eta, \eta^{\prime}\right)+F \geq 0$ to obtain the result.

ii) From Lemma $3.1 i$ ) we have

$$
\eta^{\prime \prime}-\frac{c_{1}}{\eta^{3}}+F \leq 0 .
$$

We multiply by $\eta^{\prime}$ to end the proof.

Let $D_{1}$ and $D_{2}$ be defined by

$$
D_{1}:=\left(\frac{c_{1}}{F}\right)^{1 / 3}
$$

and

$$
D_{2}:=2 \max \left\{\eta_{1}^{0}, D_{1}, \frac{1}{F}\left(\frac{1}{2}\left(\eta_{2}^{0}\right)^{2}+F \eta_{1}^{0}+\frac{c_{1}}{2\left(\eta_{1}^{0}\right)^{2}}\right), \frac{1}{F}\left(\frac{1}{2} V_{2}^{2}+F D_{1}+\frac{c_{1}}{2 D_{1}^{2}}\right)\right\} .
$$

Proposition 4.3.

$$
\eta(t)<D_{2} \quad \forall t \in[0, T[.
$$

Proof. Suppose the result is false and denote by $t_{3}>0$ the first time such that

$$
\eta\left(t_{3}\right)=D_{2} \text {. }
$$

Then, it follows that $\eta^{\prime}\left(t_{3}\right)>0$ or $\eta^{\prime}\left(t_{3}\right)=0$. In the last case, since $\eta\left(t_{3}\right)>D_{1}$, Lemma $3.1 i$ ) implies

$$
\eta^{\prime \prime}\left(t_{3}\right)=G\left(\eta\left(t_{3}\right), 0\right)<0 .
$$

So, in both cases, since $\eta \in C^{2}$, there exists $t_{1} \in\left[0, t_{3}[\right.$ such that

$$
\eta^{\prime}(t) \geq 0, \quad \forall t \in\left[t_{1}, t_{3}\right],
$$

where $t_{1}$ is the smallest number with this property. Two options concerning $t_{1}$ are possible:

Option 1: $t_{1}=0$. In this case, we have

$$
\eta^{\prime}(t) \geq 0, \quad \forall t \in\left[0, t_{3}\right] .
$$

From Lemma $4.2 i i)$ we obtain

$$
E_{2}\left(\eta\left(t_{3}\right), \eta^{\prime}\left(t_{3}\right)\right) \leq E_{2}\left(\eta_{1}^{0}, \eta_{2}^{0}\right),
$$


which implies

$$
\eta\left(t_{3}\right) \leq \frac{1}{F}\left(\frac{1}{2}\left(\eta_{2}^{0}\right)^{2}+F \eta_{1}^{0}+\frac{c_{1}}{2\left(\eta_{1}^{0}\right)^{2}}\right)
$$

and contradicts (4.2).

Option 2: $\left.t_{1} \in\right] 0, t_{3}[$.

We have in this case

$$
\eta^{\prime}\left(t_{1}\right)=0, \quad \eta^{\prime}(t) \geq 0 \quad \forall t \in\left[t_{1}, t_{3}\right]
$$

which implies

$$
\eta^{\prime \prime}\left(t_{1}\right) \geq 0
$$

From Lemma $3.1 i$ ) we obtain

$$
\frac{c_{1}}{\eta^{3}\left(t_{1}\right)} \geq F, \quad \text { that is, } \quad \eta\left(t_{1}\right) \leq D_{1} .
$$

Let $t_{2} \in\left[t_{1}, t_{3}\right]$ be a time such that

$$
\eta\left(t_{2}\right)=D_{1} .
$$

From Lemma 4.2 ii) and Proposition 4.1 we have

$$
E_{2}\left(\eta\left(t_{3}\right), \eta^{\prime}\left(t_{3}\right)\right) \leq E_{2}\left(\eta\left(t_{2}\right), \eta^{\prime}\left(t_{2}\right)\right) \leq \frac{1}{2} V_{2}^{2}+F D_{1}+\frac{c_{1}}{2 D_{1}^{2}},
$$

which implies

$$
\eta\left(t_{3}\right) \leq \frac{1}{F}\left(\frac{1}{2} V_{2}^{2}+F D_{1}+\frac{c_{1}}{2 D_{1}^{2}}\right) .
$$

This is in contradiction with (4.2) and the proof ends.

We define $V_{3}$ as follows:

$$
V_{3}:=\max \left\{1-\eta_{2}^{0}, 2 \sqrt{2 F D_{2}}, 2 \sqrt{\left(\eta_{2}^{0}\right)^{2}+2 F \eta_{1}^{0}}\right\} .
$$

Proposition 4.4.

$$
\eta^{\prime}(t)>-V_{3}, \quad \forall t \in[0, T[.
$$

Proof. Suppose the result is false and denote by $\left.t_{2} \in\right] 0, T[$ the first time such that

$$
\eta^{\prime}\left(t_{2}\right)=-V_{3}
$$

We have two options:

Option I. $\eta^{\prime}(t) \leq 0, \quad \forall t \in\left[0, t_{2}\right]$.

From Lemma $4.2 i$ ) we have

$$
E_{1}\left(\eta\left(t_{2}\right), \eta^{\prime}\left(t_{2}\right)\right) \leq E_{1}\left(\eta_{1}^{0}, \eta_{2}^{0}\right)
$$

which implies

and contradicts (4.4).

$$
\frac{1}{2}\left|\eta^{\prime}\left(t_{2}\right)\right|^{2} \leq \frac{1}{2}\left(\eta_{2}^{0}\right)^{2}+F \eta_{1}^{0}
$$

Option II: There exists $\left.t_{1} \in\right] 0, t_{2}[$ such that

$$
\eta^{\prime}\left(t_{1}\right)=0 \quad \text { and } \quad \eta^{\prime}(t) \leq 0 \quad \forall t \in\left[t_{1}, t_{2}\right]
$$


Then

$$
E_{1}\left(\eta\left(t_{2}\right), \eta^{\prime}\left(t_{2}\right)\right) \leq E_{1}\left(\eta\left(t_{1}\right), 0\right),
$$

which combined with Proposition 4.3 implies

$$
\frac{1}{2}\left|\eta^{\prime}\left(t_{2}\right)\right|^{2} \leq F D_{2}
$$

and contradicts 4.4)

The most difficult part is to obtain a lower bound of $\eta$ (Proposition 4.5). Before we remarked that from Lemma 3.7] we have

$$
\left.\left.G(\beta, \gamma) \geq c_{3} \beta^{-s_{1}}-c_{4} \gamma \beta^{-1-s_{2}}-F, \quad \forall \beta \in\right] 0, \beta_{0}\right], \quad \forall \gamma \leq 0,
$$

where

and

$$
s_{1}= \begin{cases}2\left(1-\frac{1}{\alpha}\right) & \text { in Case I (line contact) } \\ 2-\frac{3}{\alpha} & \text { in Case II (point contact) }\end{cases}
$$

$$
s_{2}= \begin{cases}2-\frac{3}{\alpha} & \text { in Case I } \\ 2-\frac{4}{\alpha} & \text { in Case II. }\end{cases}
$$

Notice that, from assumptions on $\alpha$ we deduce $s_{1}>0$ and $s_{2} \geq 0$. Let $D_{3}$ and $D_{4}>0$ be defined by

$$
\begin{gathered}
D_{3}:=\frac{2}{3} \min \left\{\eta_{1}^{0},\left(\frac{c_{3}}{F}\right)^{1 / s_{1}}\right\} \\
D_{4}:=\frac{1}{2} \min \left\{\eta_{1}^{0}, \beta_{0},\left(\frac{c_{3}}{F}\right)^{1 / s_{1}}, D_{3} \exp \left(-V_{3} D_{3}^{s_{2}} / c_{4}\right)\right\} .
\end{gathered}
$$

Proposition 4.5. Under the assumption

$$
\left\{\begin{array}{l}
\alpha \geq \frac{3}{2} \quad \text { in Case I (line contact) } \\
\text { or } \\
\alpha \geq 2 \quad \text { in Case II (point contact) }
\end{array}\right.
$$

we have $\eta(t)>D_{4}, \quad \forall t \in[0, T[$.

Proof. Suppose the result is false and denote by $\left.t_{2} \in\right] 0, T[$ the first time such that

$$
\eta\left(t_{2}\right)=D_{4} \text {. }
$$

Notice that $\eta_{1}^{0}>D_{3}>D_{4}$. Let $\left.t_{1} \in\right] 0, t_{2}[$ be the last point where

$$
\eta\left(t_{1}\right)=D_{3} .
$$

By definition of $D_{3}$ we have:

$$
\left\{\begin{array}{l}
\eta^{\prime}\left(t_{1}\right) \leq 0, \quad \eta^{\prime}\left(t_{2}\right) \leq 0 \\
c_{3} \eta(t)^{-s_{1}}>F, \quad \forall t \in\left[t_{1}, t_{2}\right]
\end{array}\right.
$$

We first see that

$$
\eta^{\prime}(t) \leq 0, \quad \forall t \in\left[t_{1}, t_{2}\right] .
$$

Suppose that (4.14) is false. Then there exists $\tau \in] t_{1}, t_{2}[$ such that

$$
\eta^{\prime}(\tau)>0
$$


Let $\tau_{1}$ be the supremum of $\left.\tau \in\right] t_{1}, t_{2}$ [ satisfying $\eta^{\prime}(\tau)>0$. It is clear that $\tau_{1}<t_{2}$ and it is a local maximum of $\eta$, which implies

$$
\left\{\begin{array}{l}
\eta^{\prime}\left(\tau_{1}\right)=0 \\
\eta^{\prime \prime}\left(\tau_{1}\right) \leq 0
\end{array}\right.
$$

Then from (4.5) and (4.13) we have

$$
\eta^{\prime \prime}\left(\tau_{1}\right)=G\left(\eta\left(\tau_{1}\right), 0\right) \geq \frac{c_{3}}{\eta\left(\tau_{1}\right)^{s_{1}}}-F>0,
$$

which contradicts (4.15). Then (4.14) is proved.

Combining (4.5) and (4.13) we deduce

$$
\eta^{\prime \prime} \geq-c_{4} \eta^{\prime} \eta^{-1-s_{2}} \geq-\frac{c_{4}}{D_{3}^{s_{2}}} \eta^{\prime} \eta^{-1} \quad \text { on }\left[t_{1}, t_{2}\right] .
$$

We integrate (4.16) to obtain

$$
\eta^{\prime}(t) \geq \eta^{\prime}\left(t_{1}\right)+\frac{c_{4}}{D_{3}^{s_{2}}} \log \left(\frac{1}{\eta(t)}\right)-\frac{c_{4}}{D_{3}^{s_{2}}} \log \left(\frac{1}{\eta\left(t_{1}\right)}\right), \quad \forall t \in\left[t_{1}, t_{2}\right],
$$

which implies

$$
\frac{c_{4}}{D_{3}^{s_{2}}} \log \left(\frac{1}{\eta\left(t_{2}\right)}\right) \leq \frac{c_{4}}{D_{3}^{s_{2}}} \log \left(\frac{1}{\eta\left(t_{1}\right)}\right)+V_{3}
$$

Then

$$
\eta\left(t_{2}\right) \geq D_{3} \exp \left(-V_{3} D_{3}^{s_{2}} / c_{4}\right)
$$

which contradicts (4.11).

Theorem 2.2 is a consequence of Propositions $4.1,4.3,4.4$ and 4.5 .

4.2. Proof of Theorem 2.5. We consider the case $h_{0} \equiv 0$.

Let us introduce the auxiliary function $w$ defined as the unique solution to the problem

$$
\begin{array}{ll}
-\Delta w=1 & \text { in } \Omega, \\
w=0 & \text { on } \partial \Omega
\end{array}
$$

and define the constant $C(\Omega)$ by

$$
C(\Omega)=\int_{\Omega} w(x) .
$$

By the maximum principle we have $w>0$ on $\Omega$, which implies

$$
C(\Omega)>0 .
$$

In the following, for any real number $z$ we denote $z^{+}=\max \{z, 0\}$ (positive part) and $z^{-}=-\min \{z, 0\}$ (negative part). We have the identity $z=z^{+}-z^{-}$.

Lemma 4.6. $\eta$ satisfies the following differential equation:

$$
\eta^{\prime \prime}=C(\Omega) \frac{\left(\eta^{\prime}\right)^{-}}{\eta^{3}}-F
$$


Proof. For $h_{0} \equiv 0$ the inequality (1.7) becomes

$$
\beta^{3} \int_{\Omega} \nabla q \cdot \nabla(\varphi-q) \geq-\gamma \int_{\Omega}(\varphi-q), \quad \forall \varphi \in K .
$$

The required result is a direct consequence of the following facts:

- if $\gamma \geq 0$ the solution of (4.18) is $q=0$;

- if $\gamma<0$ the solution of (4.18) is $q=-\frac{\gamma w}{\beta^{3}}$.

End of the proof of Theorem 2.5. Depending on the sign of $\eta_{2}^{0}$ we distinguish two different cases:

CASE $\left(\eta_{2}^{0} \leq 0\right)$. We argue by contradiction to prove

$$
\left.\eta^{\prime}(t)<0, \quad \forall t \in\right] 0, T[.
$$

Assume that (4.19) is false and let $t_{1}$ be defined as follows:

$$
t_{1}:=\inf \left\{t \in \left[0, T\left[\text { such that } \eta^{\prime}(t)=0\right\} .\right.\right.
$$

By continuity of $\eta^{\prime}$ for $\eta_{2}^{0}<0$ and by the fact that $\eta^{\prime \prime}(0)=-F$ for $\eta_{2}^{0}=0$ we have $t_{1}>0$.

By definition of $t_{1}$ and continuity of $\eta^{\prime \prime}$ we claim that

$$
\eta^{\prime \prime}\left(t_{1}\right) \geq 0 .
$$

Lemma 4.6 implies that $\eta^{\prime \prime}\left(t_{1}\right)=-F<0$, which contradicts (4.20) and proves (4.19). By (4.19) and Lemma 4.6 we deduce

$$
\eta^{\prime \prime}=-C(\Omega) \frac{\eta^{\prime}}{\eta^{3}}-F \quad \text { on } \quad[0, T[.
$$

We multiply (4.21) by $\eta^{\prime}$ to obtain

$$
\frac{d}{d t}\left[\frac{\left|\eta^{\prime}\right|^{2}}{2}+F \eta\right]=-C(\Omega) \frac{\left|\eta^{\prime}\right|^{2}}{\eta^{3}} \leq 0
$$

and deduce

which implies

$$
\frac{\left|\eta^{\prime}\right|^{2}}{2}+F \eta \leq \frac{\left|\eta_{2}^{0}\right|^{2}}{2}+F \eta_{1}^{0},
$$

$$
\eta^{\prime}>-\sqrt{\left(\eta_{2}^{0}\right)^{2}+2 F \eta_{1}^{0}} .
$$

Since $\eta$ is a nonincreasing function we deduce

$$
\eta \leq \eta_{1}^{0}, \quad t \in[0, T[.
$$

We now integrate (4.21) over $[0, t[$ to obtain

$$
\eta^{\prime}=\eta_{2}^{0}+\frac{C(\Omega)}{2 \eta^{2}}-\frac{C(\Omega)}{2\left(\eta_{1}^{0}\right)^{2}}-F t .
$$

Thanks to (4.19) and (4.22), it follows that

$$
\left.\frac{C(\Omega)}{2\left(\eta_{1}^{0}\right)^{2}}+F t-\eta_{2}^{0}-\sqrt{\left(\eta_{2}^{0}\right)^{2}+2 F \eta_{1}^{0}}<\frac{C(\Omega)}{2 \eta^{2}}<\frac{C(\Omega)}{2\left(\eta_{1}^{0}\right)^{2}}+F t-\eta_{2}^{0} \quad \text { on } \quad\right] 0, T[,
$$

which implies, for $t$ large enough, that

$$
\eta \geq \frac{C(\Omega)}{\sqrt{C(\Omega)\left(\eta_{1}^{0}\right)^{-2}+2 F t-2 \eta_{2}^{0}}}
$$


and

$$
\eta \leq \frac{C(\Omega)}{\sqrt{C(\Omega)\left(\eta_{1}^{0}\right)^{-2}+2 F t-2 \eta_{2}^{0}-\left[\left(\eta_{2}^{0}\right)^{2}+2 F \eta_{1}^{0}\right]^{\frac{1}{2}}}} .
$$

Now, (4.23), (4.19), (4.22) and (4.26) imply (2.6), while (4.26) and (4.27) imply (2.7).

CASe $\left(\eta_{2}^{0}>0\right)$. From Lemma 4.6 we deduce $\eta^{\prime \prime}=-F$ for $t \in\left[0, \frac{\eta_{2}^{0}}{F}\right]$, which implies

$$
\eta(t)=-\frac{1}{2} F t^{2}+\eta_{2}^{0} t+\eta_{1}^{0}, \quad \text { for } t \in\left[0, \frac{\eta_{2}^{0}}{F}\right] .
$$

Since $\eta^{\prime}\left(\frac{\eta_{2}^{0}}{F}\right)=0$, we may repeat the argument of the case $\eta_{2}^{0} \leq 0$ at the initial time $\frac{\eta_{2}^{0}}{F}$.

Finally, since

$$
G(\eta, 0)=-F<0, \quad \text { for any } \eta>0,
$$

we have the nonexistence of stationary solutions.

4.3. Proof of Theorem 2.7. Let us introduce the function $g:] 0,+\infty[\rightarrow \mathbb{R}$ defined by

$$
g(\beta)=G(\beta, 0) .
$$

From Lemma 3.4, it is clear that $g$ is continuous. Lemma 3.1 $i$ ) and Lemma 3.7 imply

$$
\lim _{\beta \rightarrow+\infty} g(\beta)=-F \quad \text { and } \quad \lim _{\beta \rightarrow 0} g(\beta)=+\infty,
$$

which proves the theorem.

5. Examples of nonexistence of global solutions. The goal of this section is to give some examples when $T<\infty$ for the cases when $\alpha$ does not satisfy assumption (2.5) of Theorem 2.2. Let us first give a general lemma concerning the nonexistence of global in time solutions.

Lemma 5.1. Suppose there exist positive constants $c_{a}, c_{b}, s_{3}$ and $s_{4}$ with $s_{3}, s_{4}<1$ such that

$$
G(\beta, \gamma) \leq \frac{c_{a}}{\beta^{s_{3}}}-\gamma \frac{c_{b}}{\beta^{s_{4}}}-F \quad \text { for } \beta \text { small enough and } \gamma<0 .
$$

Then, for any $\eta_{1}^{0}$ small enough and $F>0$ there exists $\eta_{2}^{0}<0$ such that $T<\infty$.

Proof. There exists $t_{1}>0$ such that $\eta^{\prime}<0$ on $\left[0, t_{1}\left[\right.\right.$, and we denote by $t_{1} \leq T$ the largest value with this property. The problem (1.6) can be expressed as a first-order system of ODEs (with $\eta, \eta^{\prime}$ denoted by $\eta_{1}, \eta_{2}$ ):

$$
\left\{\begin{array}{l}
\eta_{1}^{\prime}=\eta_{2} \\
\eta_{2}^{\prime}=G\left(\eta_{1}, \eta_{2}\right)
\end{array}\right.
$$

Since the application

$$
t:\left[0, t_{1}\left[\rightarrow \eta_{1}(t) \in\left[\eta_{1}\left(t_{1}\right), \eta_{1}^{0}\right]\right.\right.
$$

is a diffeomorphism we can express the variable $\eta_{2}$ as a function of $\eta_{1}$. We then have

$$
\frac{d \eta_{2}}{d \eta_{1}}=\eta_{2}^{-1} G\left(\eta_{1}, \eta_{2}\right)
$$


that is,

$$
\eta_{2} \frac{d \eta_{2}}{d \eta_{1}}=G\left(\eta_{1}, \eta_{2}\right) \leq \frac{c_{a}}{\eta_{1}^{s_{3}}}-c_{b} \frac{\eta_{2}}{\eta_{1}^{s_{4}}}-F \leq \frac{1}{2} \frac{\eta_{2}^{2}}{\eta_{1}^{s_{4}}}+\frac{1}{2} c_{b}^{2} \frac{1}{\eta_{1}^{s_{4}}}+c_{a} \frac{1}{\eta_{1}^{s_{3}}}-F .
$$

We then deduce that

$$
\frac{d}{d \eta_{1}}\left(\eta_{2}^{2}\right)-\eta_{2}^{2} \eta_{1}^{-s_{4}} \leq c_{b}^{2} \eta_{1}^{-s_{4}}+2 c_{a} \eta_{1}^{-s_{3}}-2 F,
$$

that is,

$$
\frac{d}{d \eta_{1}}\left(\exp \left(-\frac{\eta_{1}^{1-s_{4}}}{1-s_{4}}\right) \eta_{2}^{2}\right) \leq\left(c_{b}^{2} \eta_{1}^{-s_{4}}+2 c_{a} \eta_{1}^{-s_{3}}-2 F\right) \exp \left(-\frac{\eta_{1}^{1-s_{4}}}{1-s_{4}}\right) .
$$

Integrating over $\left[\eta_{1}, \eta_{1}^{0}\right]$ we have

$$
\begin{aligned}
& \exp \left(-\frac{\left(\eta_{1}^{0}\right)^{1-s_{4}}}{1-s_{4}}\right)\left(\eta_{2}^{0}\right)^{2}-\exp \left(-\frac{\eta_{1}^{1-s_{4}}}{1-s_{4}}\right)\left(\eta_{2}\right)^{2} \\
& \quad \leq \int_{\eta_{1}}^{\eta_{1}^{0}}\left(c_{b}^{2} \tau^{-s_{4}}+2 c_{a} \tau^{-s_{3}}-2 F\right) \exp \left(-\frac{\tau^{1-s_{4}}}{1-s_{4}}\right) d \tau \\
& \quad \leq \int_{0}^{\eta_{1}^{0}}\left(c_{b}^{2} \tau^{-s_{4}}+2 c_{a} \tau^{-s_{3}}\right) d \tau=\frac{c_{b}^{2}}{1-s_{4}}\left(\eta_{1}^{0}\right)^{1-s_{4}}+\frac{2 c_{a}}{1-s_{3}}\left(\eta_{1}^{0}\right)^{1-s_{3}}
\end{aligned}
$$

Then, it follows that

$$
\eta_{2}^{2} \geq\left[\exp \left(-\frac{\left(\eta_{1}^{0}\right)^{1-s_{4}}}{1-s_{4}}\right)\left(\eta_{2}^{0}\right)^{2}-c_{b}^{2} \frac{\left(\eta_{1}^{0}\right)^{1-s_{4}}}{1-s_{4}}-2 c_{a} \frac{\left(\eta_{1}^{0}\right)^{1-s_{3}}}{1-s_{3}}\right] .
$$

It is now possible, for any $\eta_{1}^{0}>0$ to choose $\eta_{2}^{0}<0$, with $\left|\eta_{2}^{0}\right|$ large enough, such that the right-hand side of (5.2) will be larger than 1 . By continuity we have that $\eta_{2} \leq-1$; then $t_{1}=T$ and

$$
\eta^{\prime} \leq-1 \quad \text { for all } t \in[0, T[
$$

We deduce

$$
\eta(t) \leq \eta_{1}^{0}-t
$$

so necessarily $T \leq \eta_{1}^{0}$, which ends the proof.

In the following we construct some examples of $\Omega$ and $h_{0}$ such that $G$ satisfies the above lemma. It is then necessary to obtain upper bounds for $\int_{\Omega} q$. Let us consider for any positive $\beta$ the following problems:

$$
\left\{\begin{array}{l}
\text { find } \xi_{1} \in K \text { such that } \\
\int_{\Omega}\left(h_{0}+\beta\right)^{3} \nabla \xi_{1} \nabla\left(\varphi-\xi_{1}\right) \geq \int_{\Omega} h_{0} \frac{\partial}{\partial x_{1}}\left(\varphi-\xi_{1}\right) \quad \text { for all } \varphi \in K,
\end{array}\right.
$$

and

$$
\left\{\begin{array}{l}
\text { find } \xi_{2} \in K \text { such that } \\
\int_{\Omega}\left(h_{0}+\beta\right)^{3} \nabla \xi_{2} \nabla\left(\varphi-\xi_{2}\right) \geq \int_{\Omega}\left(\varphi-\xi_{2}\right) \quad \text { for all } \varphi \in K .
\end{array}\right.
$$

Now for any $\gamma \leq 0$ we introduce the function $\xi$ given by

$$
\xi:=\xi_{1}-\gamma \xi_{2} \in K
$$


We easily see that $\xi$ satisfies

$$
\int_{\Omega}\left(h_{0}+\beta\right)^{3} \nabla \xi \nabla \psi \geq \int_{\Omega}\left(h_{0} \frac{\partial \psi}{\partial x_{1}}-\gamma \psi\right) \quad \text { for all } \psi \in K .
$$

From Kinderlehrer-Stampacchia [12] (Theorem 6.4) we deduce

$$
q \leq \xi_{1}-\gamma \xi_{2}, \quad \text { for } \beta>0, \gamma \leq 0,
$$

where $q$ is the solution to (1.7).

CASE I (Line contact). Let us consider $\Omega:=]-1,1\left[{ }^{2}\right.$ and $h_{0}\left(x_{1}, x_{2}\right)=\left|x_{1}\right|^{\alpha}$, with $\alpha>0$.

The following two lemmas give upper bounds for $\int_{\Omega} \xi_{2}$ and $\int_{\Omega} \xi_{1}$, respectively.

Lemma 5.2. There exists $c_{6}>0$ such that

$$
\begin{gathered}
0 \leq \int_{\Omega} \xi_{2} \leq c_{6} \beta^{3\left(\frac{1}{\alpha}-1\right)} \quad \text { for } \quad \alpha>1, \\
\int_{\Omega} \xi_{2} \leq c_{6} \log \frac{1}{\beta} \quad \text { for } \quad \alpha=1, \\
\int_{\Omega} \xi_{2} \leq c_{6} \quad \text { for } \quad \alpha<1 .
\end{gathered}
$$

Proof. We consider $\bar{\xi}_{2}$ to be the solution to

$$
\left\{\begin{array}{c}
\left.-\left[\left(h_{0}+\beta\right)^{3} \bar{\xi}_{2}^{\prime}\right]^{\prime}=1 \quad \text { in }\right]-1,1[ \\
\bar{\xi}_{2}(-1)=\bar{\xi}_{2}(1)=0 .
\end{array}\right.
$$

Notice that the natural extension of $\bar{\xi}_{2}$ to $\Omega$ defined by $\tilde{\xi}_{2}\left(x_{1}, x_{2}\right):=\bar{\xi}_{2}\left(x_{1}\right)$ satisfies (5.4) as well as the inequality $\tilde{\xi}_{2} \geq 0$ on $\partial \Omega$. Therefore $\tilde{\xi}_{2}$ is a supersolution to the problem (5.4), and this implies

$$
\int_{\Omega} \xi_{2} \leq \int_{\Omega} \tilde{\xi}_{2}=2 \int_{-1}^{1} \bar{\xi}_{2}
$$

We now proceed to compute $\int_{-1}^{1} \bar{\xi}_{2}$. By integration in (5.7) we obtain

$$
\left(h_{0}+\beta\right)^{3} \bar{\xi}_{2}^{\prime}=-x_{1}+c,
$$

where $c$ is a constant that satisfies

$$
c=\frac{\int_{-1}^{1} x_{1}\left(h_{0}+\beta\right)^{-3}}{\int_{-1}^{1}\left(h_{0}+\beta\right)^{-3}} .
$$

By symmetry we deduce $c=0$. Since

$$
\int_{-1}^{1} \bar{\xi}_{2}=-\int_{-1}^{1} x_{1} \bar{\xi}_{2}^{\prime}
$$

we have

$$
\int_{-1}^{1} \bar{\xi}_{2}=\int_{-1}^{1} \frac{x_{1}^{2}}{\left(\left|x_{1}\right|^{\alpha}+\beta\right)^{3}}=2 \int_{0}^{1} \frac{x_{1}^{2}}{\left(x_{1}^{\alpha}+\beta\right)^{3}} .
$$


For $\alpha>1$ we take the change of variable $x_{1}=\beta^{\frac{1}{\alpha}} y$ to obtain

$$
\int_{-1}^{1} \bar{\xi}_{2}=2 \beta^{\frac{3}{\alpha}-3} \int_{0}^{\beta^{-\frac{1}{\alpha}}} \frac{y^{2}}{\left(1+y^{\alpha}\right)^{3}} d y
$$

and the result is obtained in this case with

$$
c_{6}=2 \int_{0}^{\infty} \frac{y^{2}}{\left(1+y^{\alpha}\right)^{3}} d y<\infty .
$$

For $\alpha=1$ the result is easy to obtain by using the equality $x_{1}^{2}=\left(x_{1}+a\right)^{2}-2 a\left(x_{1}+a\right)+a^{2}$ in (5.8). For $\alpha<1$, we simply observe that $\int_{-1}^{1} \bar{\xi}_{2} \leq 2 \int_{0}^{1} x_{1}^{2-3 \alpha}<\infty$.

LEMma 5.3. There exists $c_{7}>0$ such that

$$
\begin{aligned}
& \int_{\Omega} \xi_{1} \leq c_{7} \beta^{2\left(\frac{1}{\alpha}-1\right)} \text { for } \alpha>1, \\
& \int_{\Omega} \xi_{1} \leq c_{7} \log \frac{1}{\beta}, \text { for } \alpha=1, \\
& \int_{\Omega} \xi_{1} \leq c_{7} \text { for } \alpha<1 .
\end{aligned}
$$

Proof. Let us consider $\bar{\xi}_{1}:[-1,0] \rightarrow \mathbb{R}$ to be the solution to the problem

$$
\left.-\left[\left(h_{0}+\beta\right)^{3} \bar{\xi}_{1}^{\prime}\right]^{\prime}=-h_{0}^{\prime} \quad \text { in }\right]-1,0[
$$

with the boundary conditions

$$
\begin{gathered}
\bar{\xi}_{1}(-1)=0, \\
\bar{\xi}_{1}^{\prime}(0)=0 .
\end{gathered}
$$

Integrating (5.9) we obtain

$$
\left(h_{0}+\beta\right)^{3} \bar{\xi}_{1}^{\prime}=h_{0}-c
$$

and we obtain $c=0$ from (5.11). It is clear that $\bar{\xi}_{1}^{\prime} \geq 0$ and therefore $\bar{\xi}_{1} \geq 0$. We construct $\hat{\xi}_{1}: \Omega \rightarrow \mathbb{R}$ by symmetry in $x_{1}$ :

$$
\hat{\xi}_{1}\left(x_{1}, x_{2}\right):=\left\{\begin{array}{l}
\left.\bar{\xi}_{1}\left(x_{1}\right) \quad \text { if } x_{1} \in\right]-1,0\left[\text { for } x_{2} \in\right]-1,1[, \\
\bar{\xi}_{1}\left(-x_{1}\right) \text { if } x_{1} \in\left[0,1\left[\text { for } x_{2} \in\right]-1,1[.\right.
\end{array}\right.
$$

Notice that $\hat{\xi}_{1} \geq 0$ in $\partial \Omega$ and $\hat{\xi}_{1}$ is a supersolution to the problem (5.4), so

$$
\xi_{1} \leq \hat{\xi}_{1}
$$

By symmetry, we have

Since

$$
\int_{\Omega} \xi_{1} \leq \int_{\Omega} \hat{\xi}_{1}=4 \int_{-1}^{0} \bar{\xi}_{1} .
$$

$$
\int_{-1}^{0} \bar{\xi}_{1}=-\int_{-1}^{0} x_{1} \bar{\xi}_{1}^{\prime}=-\int_{-1}^{0} x_{1} \frac{h_{0}}{\left(h_{0}+\beta\right)^{3}}=\int_{0}^{1} \frac{x_{1}^{\alpha+1}}{\left(x_{1}^{\alpha}+\beta\right)^{3}},
$$

the proof ends by following the computations of Lemma 5.2

CASE II (Point contact). Let us suppose that $\Omega:=\left\{x=\left(x_{1}, x_{2}\right) \in \mathbb{R}^{2}\right.$ such that $|x|<$ $1\}$, where we denote $|x|=\sqrt{x_{1}^{2}+x_{2}^{2}}$. We also consider $h_{0}(x)=|x|^{\alpha}$, with $\alpha>0$. 
We have the following lemmas.

LEMma 5.4. There exists $c_{8}>0$ such that

$$
\begin{aligned}
& 0 \leq \int_{\Omega} \xi_{2} \leq c_{8} \beta^{\frac{4}{\alpha}-3} \quad \text { for } \quad \alpha>\frac{4}{3} \\
& \int_{\Omega} \xi_{2} \leq c_{8} \log \frac{1}{\beta} \quad \text { for } \quad \alpha=\frac{4}{3} \\
& \int_{\Omega} \xi_{2} \leq c_{8} \quad \text { for } \quad \alpha<\frac{4}{3} .
\end{aligned}
$$

Proof. Let $\bar{\xi}_{2}$ be the unique solution to

$$
\left\{\begin{array}{c}
\left.-\frac{1}{\rho} \frac{\partial}{\partial \rho}\left[\rho\left(h_{0}+\beta\right)^{3} \frac{\partial}{\partial \rho} \bar{\xi}_{2}\right]=1 \quad \text { in }\right] 0,1[ \\
\bar{\xi}_{2}^{\prime}(0)=\xi_{2}(1)=0 .
\end{array}\right.
$$

Notice that, in fact, $\xi_{2}(x)=\bar{\xi}_{2}(|x|)$ and therefore

$$
\int_{\Omega} \xi_{2}=2 \pi \int_{0}^{1} \rho \bar{\xi}_{2}(\rho) d \rho
$$

In order to compute $\int_{0}^{1} \rho \bar{\xi}_{2}(\rho) d \rho$, we integrate in (5.12) to obtain

$$
\rho\left(h_{0}+\beta\right)^{3} \bar{\xi}_{2}^{\prime}=-\frac{1}{2} \rho^{2}+c
$$

and we deduce $c=0$ from $\bar{\xi}_{2}^{\prime}(0)=0$. Then

$$
2 \pi \int_{0}^{1} \bar{\xi}_{2}(\rho) \rho d \rho=-\pi \int_{0}^{1} \rho^{2} \bar{\xi}_{2}^{\prime} d \rho=\frac{\pi}{2} \int_{0}^{1} \frac{\rho^{3}}{\left(\rho^{\alpha}+\beta\right)^{3}} d \rho .
$$

Proceeding as in Lemma 5.2 we deduce the result.

LEMmA 5.5. There exists $c_{9}>0$ such that

$$
\begin{aligned}
& \int_{\Omega} \xi_{1} \leq c_{9} \beta^{\frac{3}{\alpha}-2} \text { for } \alpha>\frac{3}{2} \\
& \int_{\Omega} \xi_{1} \leq c_{9} \log \frac{1}{\beta} \text { for } \alpha=\frac{3}{2}, \\
& \int_{\Omega} \xi_{1} \leq c_{9} \text { for } \alpha<\frac{3}{2} .
\end{aligned}
$$

Proof. Let us consider $\bar{\xi}_{1}:[0,1] \rightarrow \mathbb{R}$ to be the solution to the problem

$$
\left.-\frac{1}{\rho} \frac{\partial}{\partial \rho}\left[\rho\left(h_{0}+\beta\right)^{3} \frac{\partial}{\partial \rho} \bar{\xi}_{1}\right]=\alpha \rho^{\alpha-1} \quad \text { in }\right] 0,1[
$$

with the boundary conditions

$$
\bar{\xi}_{1}^{\prime}(0)=0, \quad \bar{\xi}_{1}(1)=0
$$

We integrate (5.13) to obtain

$$
\rho\left(h_{0}+\beta\right)^{3} \bar{\xi}_{1}^{\prime}=-\frac{\alpha}{\alpha+1} \rho^{\alpha+1}-c .
$$


Since $\bar{\xi}_{1}^{\prime}(0)=0$ we have $c=0$. It is clear that $\bar{\xi}_{1}^{\prime} \leq 0$ and therefore $\bar{\xi}_{1} \geq 0$.

Notice that the function $\hat{\xi}_{1}(x)=\bar{\xi}_{1}(|x|)$ is a supersolution to the problem (5.4), so

$$
\int_{\Omega} \xi_{1} \leq \int_{\Omega} \tilde{\xi}_{1}=2 \pi \int_{0}^{1} \rho \bar{\xi}_{1} d \rho .
$$

Since

$$
\int_{0}^{1} \rho \bar{\xi}_{1} d \rho=-\frac{1}{2} \int_{0}^{1} \rho^{2} \bar{\xi}_{1}^{\prime} d \rho=\frac{\alpha}{2(\alpha+1)} \int_{0}^{1} \frac{\rho^{2+\alpha}}{\left(\rho^{\alpha}+\beta\right)^{3}} d \rho
$$

the proof ends by following the computations of Lemma 5.2

From the above results we deduce that in the line contact case for $\Omega:=] 0,1\left[^{2}\right.$ and $h_{0}(x)=\left|x_{1}\right|^{\alpha}$ we have $T<\infty$ for some initial data when $\alpha<\frac{3}{2}$. Similarly, in the point contact case, $\Omega=B(0,1)$ and $h_{0}(x)=\left|x_{1}^{2}+x_{2}^{2}\right|^{\frac{\alpha}{2}}$, we have that $T<\infty$ when $\alpha<2$.

REMARK 5.6. The main difficulty, which appears to generalize the results of this section to arbitrary $\Omega$ and $h_{0}$, is to obtain an upper bound for $\int_{\Omega} q$. The arguments to obtain this upper bound must be global in $\Omega$, while for obtaining a lower bound for $q$, local arguments are sufficient (see Lemma 3.6).

\section{REFERENCES}

[1] Buscaglia G., Ciuperca I., Hafidi I., Jai M.: Existence of equilibria in articulated bearings. J. Math. Anal. Appl. 328, pp. 24-45 (2007). MR2285530 (2008c:74069)

[2] Buscaglia G., Ciuperca I., Hafidi I., Jai M.: Existence of equilibria in articulated bearings in presence of cavity. J. Math. Anal. Appl. 335, pp. 841-859 (2007). MR2345504 (2008f:76068)

[3] Ciuperca I., Hafidi I., Jai M.: Singular perturbation problems for the incompressible Reynolds equation. Electronic Journal of Differential Equations, no. 83, pp. 1-19 (2006). MR2240831(2007f:76053)

[4] Ciuperca I., Jai M., Tello J.I.: On the existence of solutions of equilibria in lubricated journal bearings. SIAM, Mathematical Analysis, 40, pp. 2316-2327 (2009). MR2481296

[5] Conca C., San Martin J.A., Tucsnak M.: Existence of solutions for the equations modelling the motion of a rigid body in a viscous fluid. Comm. Partial Differential Equations, 25, pp. 1019-1042 (2000). MR.1759801 (2001c:35199)

[6] Desjardins B., Esteban M.J.: Existence of weak solutions for the motion of rigid bodies in a viscous fluid. Arch. Ration. Mech. Anal. 146, pp. 59-71 (2000). MR.1682663(2000b:76024)

[7] Díaz J.I., Tello J.I.: A note on some inverse problems arising in lubrication theory. Differential Integral Equations, 17, pp. 583-591 (2004). MR2054936 (2005a:35278)

[8] Frêne J., Nicolas D., Deguerce B., Berthe D., Godet M.: Lubrification hydrodynamique. Eyrolles, Paris (1990).

[9] Gérard-Varet D., Hillairet M.: Regularity issues in the problem of fluid structure interaction. Arch. Ration. Mech. Anal. 195, pp. 375-407 (2010). MR2592281

[10] Hesla T.I.: Collision of smooth bodies in viscous fluids: a mathematical investigation. Ph.D. Thesis, University of Minnesota (2004).

[11] Hillairet M.: Lack of collision between solid bodies in a 2D incompressible viscous flow. Communications on PDEs 32, pp. 1345-1366 (2007). MR2354496 (2008i:76053)

[12] Kinderlehrer D., Stampacchia G.: An Introduction to Variational Inequalities and Their Applications. Academic Press, New York (1980). MR567696 (81g:49013) 